\title{
NHS-funded implants: are they as common as they seem?
}

The provision of dental implants: current practice among university and hospital specialists in restorative dentistry within the UK and Ireland.

Br Dent J 2020; 228: 39-43. https://doi.org/10.1038/s41415-019-1112-y.

Tooth loss can significantly impact an individual's quality of life by causing a great amount of aesthetic and functional discomfort. Dental implants can offer a solution for patients with tooth loss. These are mostly privately funded, with a few cases eligible for NHS-funding as per Royal College of Surgeons of England (RCSE) 2012 guidelines. However, since the last update, there haven't been any studies re-evaluating the current guidelines to reflect the changing trends in current implant practice. ${ }^{1}$

This study aims to establish current implant practice among university and hospital specialists in restorative dentistry within the United Kingdom and Ireland. Online questionnaires were sent out by email to 150 specialists with a $27 \%$ response rate (41). Seventy percent of the respondents provided implant treatment, of which $76 \%$ placed and $100 \%$ restored implants. Besides, the restorative clinicians adopted a multidisciplinary approach with oral surgeons or oral and maxillofacial surgeons as part of their implant team to do complex procedures such as bone grafting, sinus lifting etc.

NHS-funded implant cases vary among local NHS trusts since it is determined by the needs of the local population and the funding available. The respondents agreed funding is prioritised for individuals affected with hypodontia, malignancy \& oro-facial trauma. These conditions have overtaken denture intolerance to be the most eligible for funding as shown by a previous study in 2001. This suggests there has either been a decline in funding for denture intolerance or that other groups have been prioritised instead.

The RCSE guidelines include assessing the medical, social and dental history before treatment, since it may have an impact on the prognosis of the implant. The respondents agreed on important medical factors which should be considered including irradiation, smoking, and bisphosphonates; this may be due to the poor prognosis among smokers and the risk of jaw necrosis among patients with irradiation or on bisphosphonates. Age and stress were rated as the least important factors. There's a strong agreement that implants are contraindicated in the presence of dental factors including untreated periodontitis, poor $\circlearrowleft$ oral hygiene, uncontrolled caries and interocclusal space.

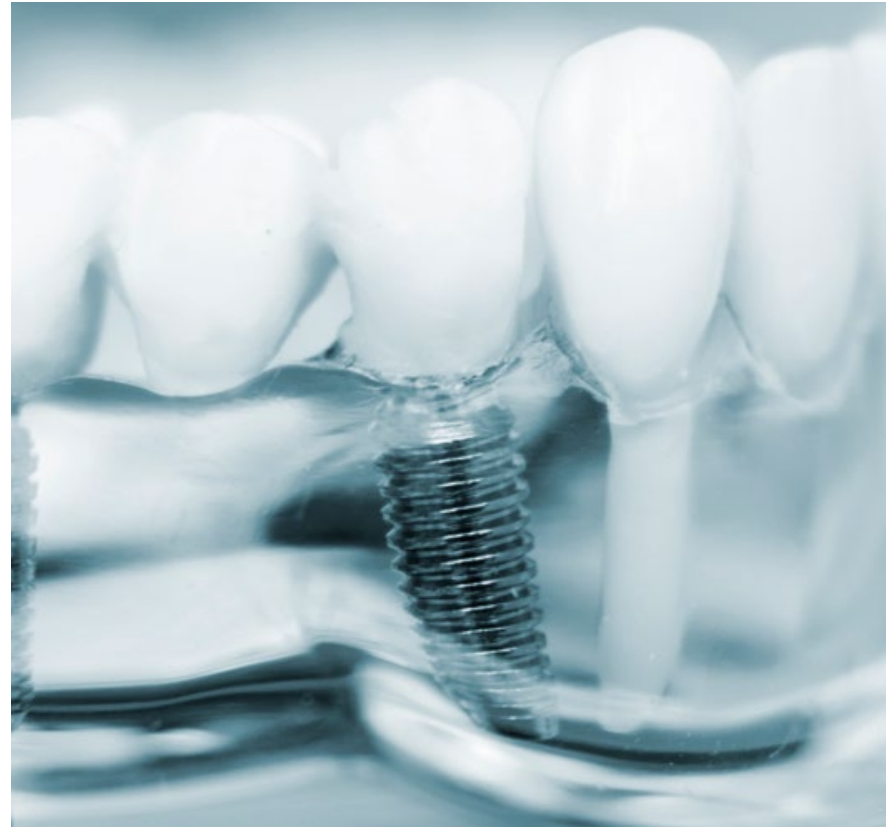

Despite the poor response, the authors acknowledged that different methods such as interviews, and telephone and postal questionnaires, may have been more appropriate than an online survey. Furthermore, the study considers the results may be unreliable due to the low number of respondents and the risk of participant bias.

While the study is promising, further studies would be valuable in recognising the current use of RCSE guidelines for NHS-funding, and how it differs among trusts while also looking at the population needs. By Anesah Anwar, King's College London BDS4

\section{References}

1. Royal College of Surgeons. Guidance on the standards of care for NHS-funded dental implant treatment. Updated 2019. Available at https://www.rcseng.ac.uk/dental-faculties/ $\mathrm{fds/publications-guidelines/clinical-guidelines/} \mathrm{(accessed} \mathrm{January} \mathrm{2020).}$

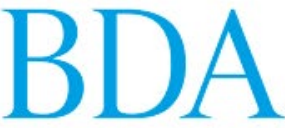

British Dental Association

\section{Managing failures in restorative dentistry}

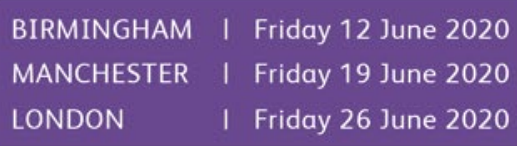

Book now:

bda.org/seminars

02075634590 | events@bda.org
Diagnosis,

management and

prevention of failures

Louis Mackenzie

A] Ray-Chaudhuri

5 hours CPD

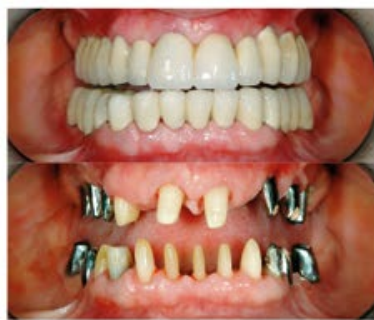

Sponsored by

\section{Seminar Series}

